\title{
Extending the Building Cube Method to Curvilinear Mesh with Adaptive Mesh Refinement
}

\author{
Xinrong $\mathrm{SU}^{*}$, Satoru YAMAMOTO ${ }^{* *}$ and Kazuhiro NAKAHASHI ${ }^{* * *}$ \\ * Key Laboratory for Thermal Science and Power Engineering of Ministry of Education, Tsinghua University \\ Haidian District, Beijing, 100084, P. R. China \\ E-mail: suxr@mail.tsinghua.edu.cn \\ ** Department of Computer and Mathematical Sciences, Tohoku University \\ 6-6-01 Aoba, Aramaki, Aoba-ku, Sendai 980-8579, Japan \\ *** Japan Aerospace Exploration Agency, Chofu Aerospace Center \\ 7-44-1 Jindaiji Higashi-machi, Chofu-shi, Tokyo 182-8522, Japan
}

Received 24 February 2014

\begin{abstract}
Building Cube Method (BCM) adopts block-structured Cartesian mesh and finer resolution can be used where the flow contains detailed flow structures. This paper reports the recent progress in extending BCM to curvilinear body-fitted mesh with Adaptive Mesh Refinement (AMR). Compared to Cartesian mesh based AMR, body-fitted AMR strategy is more complicated and less studied. In this paper the key components of body-fitted curvilinear mesh based AMR are introduced. A pressure based refinement criterion is used to detect both shock wave and strong vortex. Cubic interpolation is used to preserve the quality of the refined mesh. A sub-block based refinement strategy is also developed for the treatment of singular geometry features. Combined with the high order scheme, an accurate and robust curvilinear mesh based AMR tool is developed with which the flow details can be automatically captured. Numerical examples are given to validate the numerical properties of current AMR tool and to demonstrate the benefits of current body-fitted AMR strategy.
\end{abstract}

Key words : BCM, AMR, Multi-block structured curvilinear mesh, Shock wave, Vortical flow, Accuracy, Robustness

\section{Introduction}

Computational Fluid Dynamics (CFD) eventually plays a vital role in both research and industrial communities. Continuous efforts are made towards more accurate results by designing higher accuracy numerical methods and better meshing strategies. Of all the difficulties, the time elapsed during the mesh generation increases dramatically relative to the time needed for the solution of governing equations on the modern high performance computers. Cartesian meshes, especially the block structured Cartesian mesh are easy to generate and adapt. The block based mesh topology naturally supports various high order numerical methods. The generation of Cartesian mesh can be made fully automatic and robust. The BCM approach developed by Nakahashi $(2005,2006)$ represents a typical Cartesian mesh based simulation system.

In the CFD simulation, there are many important flow structures and the accurate prediction of them requires proper mesh resolution. On the other hand, the mesh density is always determined based on experience or rough estimation. So we have to improve the mesh and rerun the CFD solver. This process may be repeated several times and it adds a large amount of labor work. From the seminal work of Berger and Oliger (1984), Berger and Colella (1989), AMR tool can be used to relieve this burden by automatically adapting the mesh resolution to local flow structure. With AMR the costly global mesh refinement can be avoided and the balance between accuracy and cost can be achieved. Cartesian mesh based AMR is very easy as the mesh enrichment is conducted in the Cartesian space and there are several mature Cartesian AMR systems widely used in the fields of combustion and astrophysics, such as PARAMESH (MacNeice, 2000).

Curvilinear mesh based AMR is much difficult, as all the mesh manipulations are conducted in the curvilinear space. Berger and Jameson (1985) reported their body fitted AMR tool and the numerical result with two dimensional inviscid 
case. Groth et al. (1999) developed a curvilinear AMR tool for magneto-hydrodynamics and their method was tested with several cases with simple geometries. Jouhaud et al. (2005) developed a structured mesh AMR strategy with multigrid acceleration and reported the results for airfoil cases. Matsuo et al. (2012) developed a parallel structured AMR tool and demonstrated several numerical results for re-entry capsule and coaxial jet flow. To sum up, the body-fitted AMR is less studied and used compared to the Cartesian AMR and in existing studies the AMR tool was mostly tested with simple geometry and smooth mesh, which is far from the real application. In the work of Buning and Pulliam (2012) they summarized the current status of body-fitted AMR and also discussed various existing challenges, especially the stable and efficient handling of non-smooth and singular mesh.

In this work we are interested in extending the Cartesian mesh based BCM framework to support body-fitted AMR tool and special cares are taken on the stable and efficient handling of non-smooth and singular geometry features. Current AMR tool is based on the tree data structure of the BCM method. A pressure based refinement function is used and theoretical analysis shows that it is able to detect both shock wave and strong vortex. This AMR tool is mainly used in high Reynolds number cases where the mesh is highly anisotropic. To preserve the smoothness and grid stretching, cubic interpolation is used for mesh refinement. For its applications with complex geometries, a big challenge is the treatment of singular features and refined mesh with negative Jacobian would be generated if this issue is not well handled. We propose a new refinement strategy where the mesh block is automatically divided into several sub-blocks and refined mesh is generated within the sub-blocks. In this manner the accuracy and robustness issues of curvilinear mesh refinement are simultaneously satisfied. Together with high accuracy scheme and efficient solution method, an efficient body-fitted AMR framework is developed. Current method is tested with a series of examples and several representing cases will be given in this paper. With these examples, the accuracy, efficiency and robustness of current approach are verified.

This paper is organized as follows: in the second section the governing equations are given; current AMR tool is detailed in the third section and the fourth section introduces the numerical method; several numerical examples are given in the fifth section and the last section concludes this paper.

\section{Governing equations and brief introduction of BCM}

The compressible Navier-Stokes equation in the conservative form can be expressed as

$$
\frac{\partial \mathbf{U}}{\partial t}+\frac{\partial\left(\mathbf{F}_{i n v}-\mathbf{F}_{v}\right)}{\partial x}+\frac{\partial\left(\mathbf{G}_{i n v}-\mathbf{G}_{v}\right)}{\partial y}+\frac{\partial\left(\mathbf{H}_{i n v}-\mathbf{H}_{v}\right)}{\partial z}=0
$$

where $\mathbf{U}$ denotes the conservative variables, $\mathbf{F}_{\text {inv }}, \mathbf{G}_{i n v}$ and $\mathbf{H}_{i n v}$ denote the convective fluxes and $\mathbf{F}_{v}, \mathbf{G}_{v}$ and $\mathbf{H}_{v}$ denote the viscous fluxes. The Spalart-Allmaras model (Spalart and Allmaras, 1992) is used to compute the turbulent viscosity.

In the BCM method, the computational domain is meshed with block structured Cartesian mesh and every mesh block is denoted as a cube. Every cube has the same number of mesh points. As given in Fig. 1, the mesh is refined by isotropically splitting the cube into children cubes and the whole process is represented with a tree based data structure. The BCM mesh generation is extremely fast. The use of structured mesh enables the application of many existing high

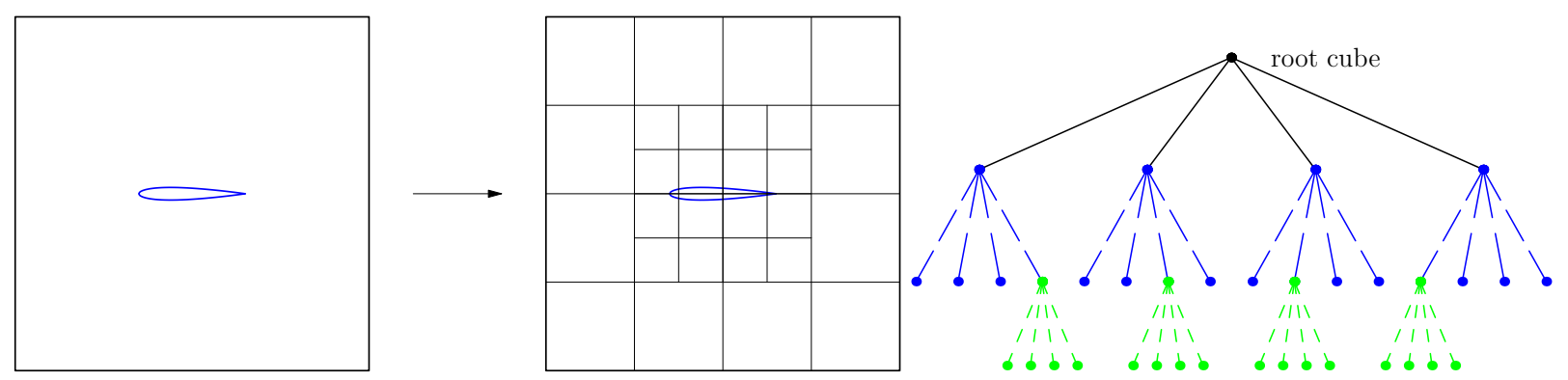

(a) Recursive division to generate children cubes

(b) Tree data structure of Fig. 1(a)

Fig. 1 Mesh generation process and the tree based representation in the BCM method.

order schemes. Furthermore, with the structured Cartesian mesh there is no need to compute and store the mesh metrics and this is a big saving in CPU cost and memory. For the tree based BCM data structure, perfect load balance and high parallel efficiency can be achieved with techniques like the space filling curve.

Although the BCM has many advantages, there are also several drawbacks. First, the meshlines are not wall-aligned and special wall treatment method is required. Second, during the mesh generation process, the BCM mesh resolution is 
not adapted to the length scale of local flow structures, but the local geometry. In this work we are interested in extending the functionality of the BCM to support both body-fitted mesh and AMR. All key elements of the BCM method form the basis of this work and now every cube in Fig. 1(b) may represent a Cartesian mesh block or a body-fitted mesh block. As explained in Section 3.2, for every body-fitted mesh block, a corresponding Cartesian mesh block is also generated and the mappings between the Cartesian space and the curvilinear space are used in the curvilinear refinement.

\section{Curvilinear mesh based AMR}

The flowchart of CFD simulation is given in Fig. 2. After generating the mesh, the solution process is conducted.

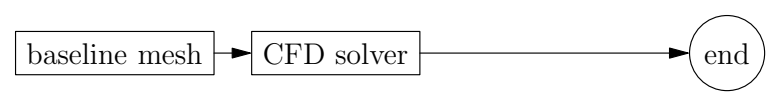

Fig. 2 Flowchart of CFD simulation without AMR.

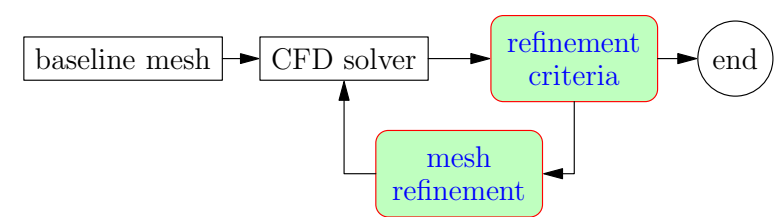

Fig. 3 Flowchart of AMR based CFD simulation.

According to the numerical results the baseline mesh may be manually regenerated, which represents a time-consuming part in the simulation process. For comparison purpose, the flowchart of AMR based CFD simulation is demonstrated in Fig. 3. Within the AMR system the mesh regeneration is automatically conducted and two key elements are added, including the refinement criteria and the mesh refinement. The refinement criterion is used to automatically mark the region requiring denser mesh. The mesh refinement should also be automatically conducted to relieve the burden of manual mesh manipulation. Their implementations will be detailed in the following.

\subsection{Refinement criteria}

For typical aerospace applications, there are several types of important flow features, such as the boundary layer, the shock wave and the strong vortex. Mesh refinement mostly takes place in these regions. With huge amount of existing knowledge about the boundary layer, the mesh density in the wall-normal direction can be predetermined quite well which gives appropriate $y^{+}$distribution. Also the streamwise mesh density can be determined to limit the maximum aspect ratio, following some theoretical analysis (Mavriplis, 2003; Su et al., 2013a). As a result the mesh density in the boundary layer can always be adequate. For the other two types of important flow features, in the literature there are two kinds of refinement criteria. The density or pressure gradient based function can be used to identify the shock wave. For strong vortex flow, vorticity based parameters, such as the vorticity magnitude and the Q-criterion are preferred.

A pressure based refinement criterion is used to detect both shock wave and strong vortex. The shock sensor function used in the famous Jameson-Schmidt-Turkel central scheme (Jameson et al., 1981) is computed along the grid line as

$$
\psi_{i}=\frac{\left|p_{i+1, j, k}-2 p_{i, j, k}+p_{i-1, j, k}\right|}{p_{i+1, j, k}+2 p_{i, j, k}+p_{i-1, j, k}}, \psi_{j}=\frac{\left|p_{i, j+1, k}-2 p_{i, j, k}+p_{i, j-1, k}\right|}{p_{i, j+1, k}+2 p_{i, j, k}+p_{i, j-1, k}}, \psi_{k}=\frac{\left|p_{i, j, k+1}-2 p_{i, j, k}+p_{i, j, k-1}\right|}{p_{i, j, k+1}+2 p_{i, j, k}+p_{i, j, k-1}}
$$

and then the refinement function is simply computed as

$$
\psi_{p}=\max \left(\psi_{i}, \psi_{j}, \psi_{k}\right)
$$

From Eq. 2 it is clear $\psi_{p}$ has a large value in the shock wave region. Recent research (Su and Yamamoto, 2014) found that the function $\psi_{p}$ is also able to detect strong vortex. For simplicity, consider the vortex given in Fig. 4 where the velocity is expressed in the $(r, \theta, z)$ coordinate and it is common knowledge that pressure reaches its minima in the vortex center. This phenomenon can be explained by the simplified momentum equation expressed in the $(r, \theta, z)$ coordinate:

$$
\frac{\partial p}{\partial r}=\rho \frac{V_{\theta}^{2}}{r}
$$

For simplicity, assume the mesh lines are along the coordinate $r$, then from Eq. 2, $\psi_{p}$ can be approximated as

$$
\psi_{p} \sim \frac{(\delta r)^{2}}{p} \frac{\partial^{2} p}{\partial r^{2}}
$$

where $\delta r$ is the mesh spacing. Adopting Eq. 4 and Eq. 5 and neglecting some high order terms, a rough estimation of $\psi$ is

$$
\psi_{p} \sim \rho \frac{(\delta r)^{2}}{p} \frac{V_{\theta}^{2}}{r^{2}}
$$




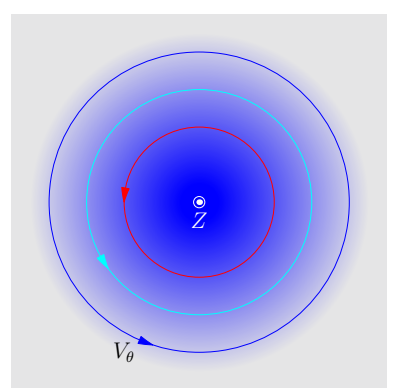

Fig. 4 Velocity and pressure distributions in the strong vortex region.

From the above equation, in the vortex core $\psi_{p}$ has a large value and this phenomenon is especially clear in strong vortex where $V_{\theta}$ is large. Also from Eq. 6, only with very dense mesh the refinement function can be reduced below the threshold value $\psi_{0}$. From the above analysis, current refinement criterion is able to detect both shock wave and strong vortex. It has clear physical meanings and is free of tunable parameter. One defect of the pressure based approach is its failure to detect the contact discontinuity which has constant pressure distribution but non-smooth density distribution that may grow into vortices. In this circumstance the pressure based criterion fails and the refinement function is improved by considering the curvatures of density and temperature. Similar as defined in Eq. 2, the following formulations are first computed

$$
\psi_{\rho, i}=\frac{\left|\rho_{i+1, j, k}-2 \rho_{i, j, k}+\rho_{i-1, j, k}\right|}{\rho_{i+1, j, k}+2 \rho_{i, j, k}+\rho_{i-1, j, k}}, \psi_{\rho, j}=\frac{\left|\rho_{i, j+1, k}-2 \rho_{i, j, k}+\rho_{i, j-1, k}\right|}{\rho_{i, j+1, k}+2 \rho_{i, j, k}+\rho_{i, j-1, k}}, \psi_{\rho, k}=\frac{\left|\rho_{i, j, k+1}-2 \rho_{i, j, k}+\rho_{i, j, k-1}\right|}{\rho_{i, j, k+1}+2 \rho_{i, j, k}+\rho_{i, j, k-1}}
$$

and then the density curvature is defined as $\psi_{\rho}=\max \left(\psi_{\rho, i}, \psi_{\rho, j}, \psi_{\rho, k}\right)$. The temperature curvature is computed in the same manner and will not be repeated here. Finally the refinement criterion is computed as $\psi=\max \left(\psi_{p}, \psi_{\rho}, \psi_{T}\right)$.

\subsection{Accurate and robust curvilinear mesh refinement}

After computing the refinement function, mesh cells of which the refinement function $\psi$ is larger than the user-defined threshold value $\psi_{0}$ are marked for refinement. Then the point clustering algorithm proposed by Berger and Rigoutsos (1991) is employed to generate a set of rectangles (cuboids in three dimensions) and a new cube will be generated inside each rectangle. This process is efficiently implemented using the tree based data structure of the BCM method. The mesh

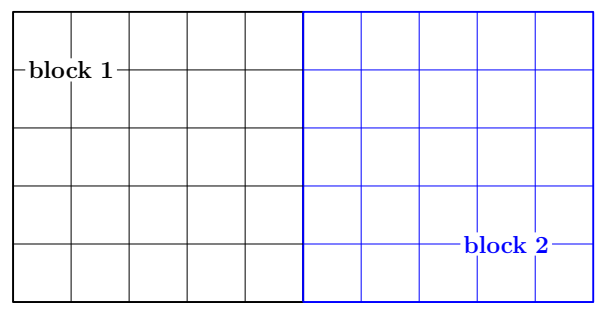

(a) the baseline mesh

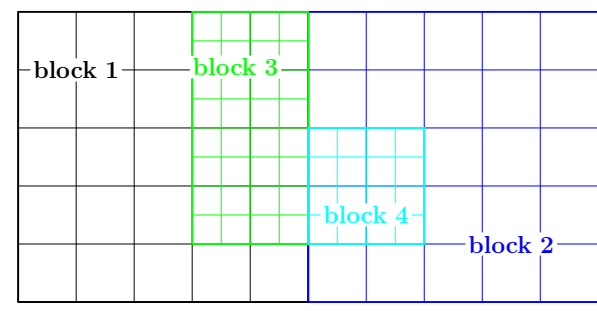

(b) after $1^{\text {st }}$ mesh refinement

Fig. 5 Demonstration of the AMR mesh refinment.

refinement expressed in Fig. 5 has to be conducted with curvilinear mesh and there are several requirements. First, mesh qualities like smoothness and aspect ratio must be preserved. Also the refinement strategy should be enough robust so as to generate a set of valid mesh suitable for CFD solver.

For turbulent flow computations, in the near wall region mesh cell is stretched with large aspect ratio. A direct and simple method is to generate finer mesh by linear interpolation. However, results show that this method does not preserve the smoothness and stretching in the near wall region, which may adversely affect the efficiency of AMR. A more complex refinement method is used here. As demonstrated in Fig. 6, the body-fitted mesh is first mapped from curvilinear to corresponding BCM mesh which is simply expressed by Cartesian formulation. Denote $x$ be the original coordinates of the mesh and $X$ be the coordinates in the Cartesian space, the mapping between these two spaces are formulated and denoted as $X=X(x)$. The mesh refinement is conducted in the Cartesian space with linear interpolation and the refinement is mapped back to the curvilinear mesh by the inverse mapping $x=x(X)$. In this work cubic formulations are used for these two mappings and the tri-cubic interpolation developed by Lekien and Marsden (2005) is adopted. As given in Fig. 6, with this strategy the smoothness and mesh stretching are better preserved. 
linear refinement
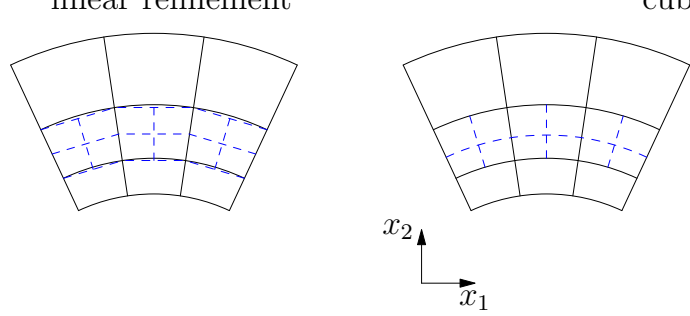

cubic refinement

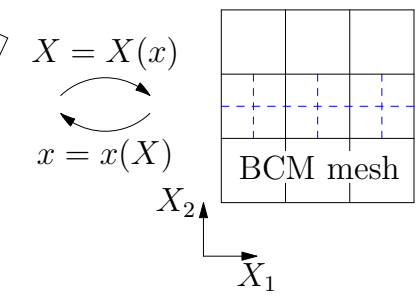

Fig. 6 Comparison of linear refinement and cubic refinement for curvilinear mesh.

Curvilinear refinement with cubic formulation successfully preserves the mesh qualities like smoothness and aspect ratio; however, it proposes a new difficulty with regard to the robustness. In real applications there may exist several singular geometry features, such as sharp corners and trailing edges. During the mesh generation, special cares are needed to preserve these important features and mesh quality near the geometrical singularity. With the cubic refinement, it would fail to preserve the exact singular feature. Even worse, in these regions cubic refinement may result in mesh tangling or mesh cells with negative Jacobian. An example about a super-critical airfoil is given in Fig. 7. As given in

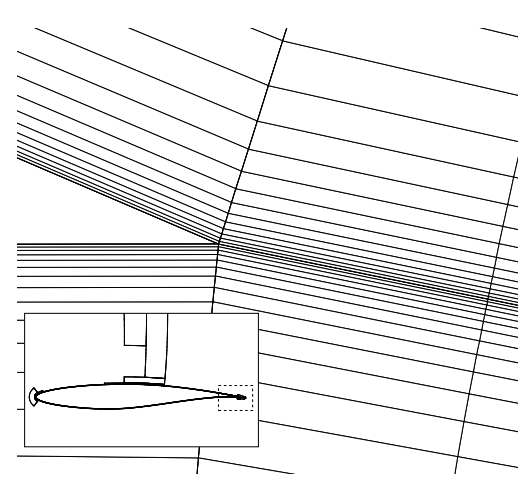

(a) the baseline mesh

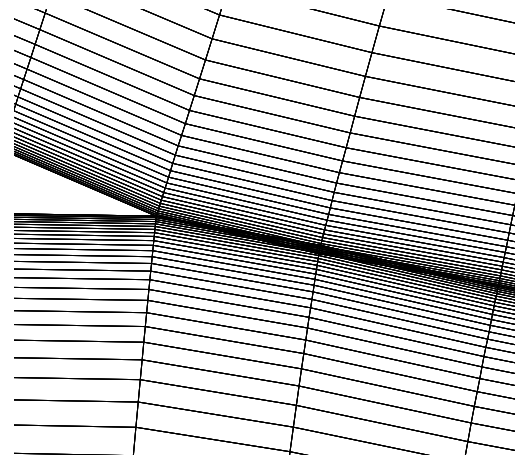

(b) refined mesh with block based refinement

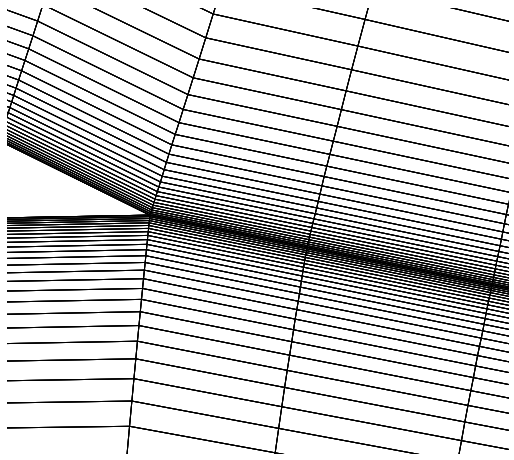

(c) refined mesh with current sub-block based refinement strategy

Fig. 7 Treatment of singular features during the curvilinear mesh refinement.

Fig. 7(b), the cells near the trailing edge are refined and after the cubic refinement, it is obvious that the mesh is not suitable for CFD solver because mesh tangling occurs near the trailing edge and this is a consequence of the singularity.

Linear refinement is free of this problem; but it does not preserve the mesh smoothness and aspect ratio. To solve this problem, a new refinement strategy is developed. Near the geometry singularity, if the mesh topology is not well designed, the mesh lines will unavoidably have abrupt changes. In this circumstance any high order interpolation will suffer from the problem of numerical oscillation and the interpolation across singularity should be avoided. Inspired by this analysis, we try to divide the block which may contain geometry singularity into several sub-blocks. As given in Fig. 8, the mesh
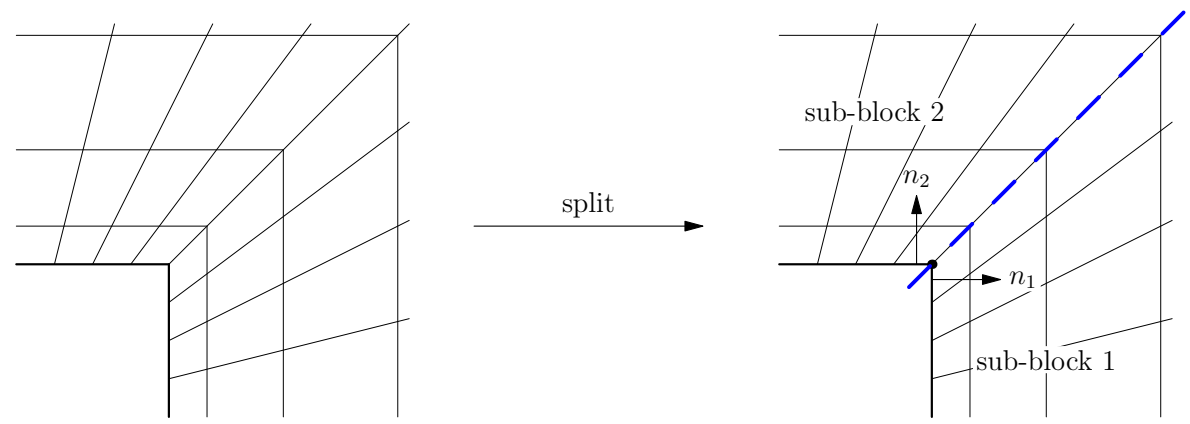

Fig. 8 Demonstration of current block-division strategy.

block is first divide into two sub-blocks by detecting the corner singularity. This process will be repeated several times until the mesh lines inside each sub-block are smooth enough. Then cubic refinement is conducted inside the sub-block. In this manner the requirements of preserving smoothness, stretching and singular features are fully satisfied. The block 
division process can be made fully automatic as in the literature there are already several reliable methods to detect the geometrical singularities (Jiao, 2008). The block-division process consumes negligible CPU time and the cost overhead is less than $0.1 \%$, according to our tests. Fig. 7 (c) gives the refined mesh with current sub-block based strategy. Compared to Fig. 7(b), the problem of mesh tangling is fully resolved and the refined mesh is of high quality.

\section{Residual evaluation and solution}

Let $\mathbf{F}_{n}=\mathbf{F}_{\text {inv }} n_{x}+\mathbf{G}_{i n v} n_{y}+\mathbf{H}_{\text {inv }} n_{z}$ be the numerical approximation of convective flux and $\mathbf{F}_{n}^{v}=\mathbf{F}_{v} n_{x}+\mathbf{G}_{v} n_{y}+\mathbf{H}_{v} n_{z}$ be the viscous flux. In this work finite volume method is employed and the Navier-Stokes equation is semi-discretized as

$$
\begin{aligned}
& \operatorname{VOL} \frac{\partial \mathbf{U}}{\partial t}+\mathbf{F}_{n, i+1 / 2, j, k}+\mathbf{F}_{n, i, j+1 / 2, k}+\mathbf{F}_{n, i, j, k+1 / 2}-\mathbf{F}_{n, i-1 / 2, j, k}-\mathbf{F}_{n, i, j-1 / 2, k}-\mathbf{F}_{n, i, j, k-1 / 2} \\
& -\left(\mathbf{F}_{n, i+1 / 2, j, k}^{v}+\mathbf{F}_{n, i, j+1 / 2, k}^{v}+\mathbf{F}_{n, i, j, k+1 / 2}^{v}-\mathbf{F}_{n, i-1 / 2, j, k}^{v}-\mathbf{F}_{n, i, j-1 / 2, k}^{v}-\mathbf{F}_{n, i, j, k-1 / 2}^{v}\right)=0
\end{aligned}
$$

where VOL denotes the volume of the mesh cell.

In the following only $\mathbf{F}_{n, i+1 / 2, j, k}$ is discussed and for simplicity the triple subscripts $(i+1 / 2, j, k)$ is briefed as $i+1 / 2$. $\mathbf{F}_{n, i+1 / 2}$ is computed with approximated Riemann solver and has the form of $\mathbf{F}_{n}=\mathbf{F}_{n}\left(\mathbf{U}_{L}, \mathbf{U}_{R}\right)$. Without otherwise stated, throughout this work the Roe scheme is used. High order accurate reconstruction should be used to compute the left and right state variables, $\mathbf{U}_{L}$ and $\mathbf{U}_{R}$, for high order accuracy. Currently second or third order Monotone Upwind Scheme for Conservation Laws (MUSCL) method (van Leer, 1977) is widely used. For all MUSCL reconstruction strategies, the accuracy reduces to first order in the shock region. Another candidate is the Weighted Essentially Nonoscillatory (WENO) scheme (Liu et al., 1994) which offers higher than first order accuracy in the shock region. WENO is much expensive and has deteriorated convergence property due to its reduced numerical dissipation. As a result WENO is less used in the aerodynamics community until recently. Su et al. (2013b) proposed a much simplified characteristic variable based WENO method and verified its superior accuracy and better resolution of small scale flow features. In this work the fifth order WENO method proposed by Su et al. (2013b) is employed to compute $\mathbf{U}_{L}$ and $\mathbf{U}_{R}$. As given in Fig. 9, for any

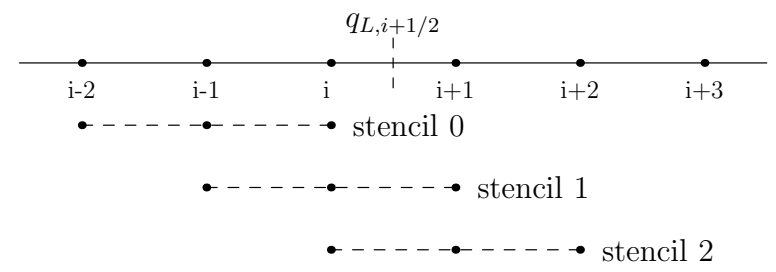

Fig. 9 Reconstruction stencils of the fifth order WENO to compute $q_{i+1 / 2}^{L}$.

physical variable $q$, the reconstructed value can be obtained as the weighted summation of three reconstruction stencils

$$
q_{i+1 / 2}^{L}=\omega_{0} q_{0}+\omega_{1} q_{1}+\omega_{2} q_{2}
$$

where

$$
q_{0}=\frac{1}{3} q_{i-2}-\frac{7}{6} q_{i-1}+\frac{11}{6} q_{i}, q_{1}=-\frac{1}{6} q_{i-1}+\frac{5}{6} q_{i}+\frac{1}{3} q_{i+1}, q_{2}=\frac{1}{3} q_{i}+\frac{5}{6} q_{i+1}-\frac{1}{6} q_{i+2}
$$

and the reconstruction weights are defined as

$$
\omega_{m}=\frac{C_{m}}{\epsilon+I S_{m}} / \sum_{n=0}^{2} \frac{C_{n}}{\epsilon+I S_{n}}, m=0,1,2
$$

where $I S_{n}$ is the smoothness indicator defined by the variation of the variable. $q_{i+1 / 2}^{R}$ can be constructed in a symmetric manner and will not be repeated here. In this work $q$ is taken to be the characteristic variable and the WENO reconstruction is conducted in the characteristic space. After that $\mathbf{U}_{L}$ and $\mathbf{U}_{R}$ are recovered by the inverse transformation. With the adoption of the fifth order WENO and AMR strategy, the solver performs superior than existing tools which use the baseline mesh and second order MUSCL scheme. An implicit time marching method as depicted in Su et al. (2013b) is used and multigrid method is also used to accelerate the convergence speed.

\section{Results and discussion}

Current BCM based body-fitted AMR tool is tested with a series of external and internal examples and several of them will be given here. 


\subsection{Flow past NACA-0012 airfoil}

The first example is about the flow past the NACA-0012 airfoil. The freestream flow is subsonic and the Mach number is $M a_{\infty}=0.55$ and the Reynolds number is $R e=6 \times 10^{6}$. Due to the large incident angle of $\alpha=8.34$ degrees, the flow undergoes a strong turning around the leading edge and accelerates to supersonic. A strong shock wave is thus formed in this area and a small separation bubble exists after the shock wave.

The baseline mesh is composed of $256 \times 64$ cells and is optimized for zero incident angle. The computed Mach number distributions are given in Fig. 10(a). From Fig. 10(a) the shock wave is captured; however, the shock wave profile

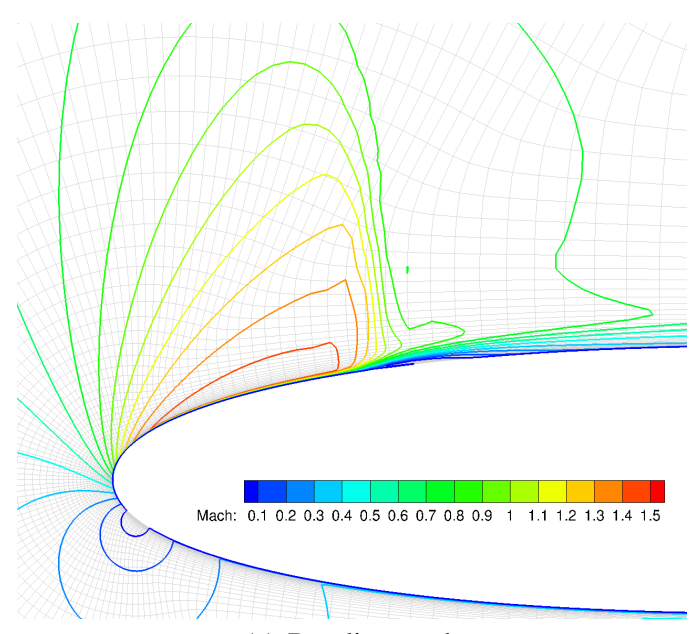

(a) Baseline mesh

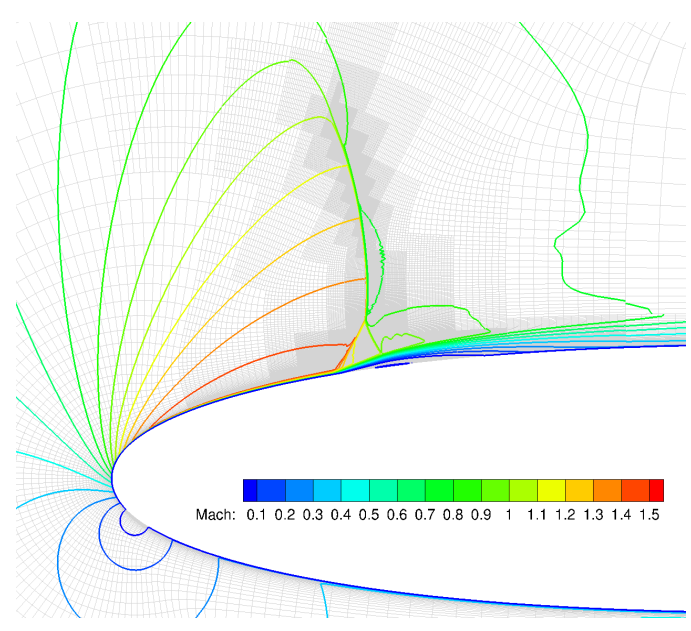

(b) 5 levels of AMR

Fig. 10 Comparison of the Mach number distributions near the leading edge, NACA-0012 test case.

spans about $2 \sim 3$ cells and flow details are totally lost. AMR is conducted for this test case and the results after 5 levels of refinement are demonstrated in Fig. 10(b). With mesh refinement, more points are automatically positioned in the shock wave area. It is clear that the shock wave has a $\lambda$ type structure, which is composed of the normal shock, the strong front foot and the weak rear shock. These details are seldom resolved by the baseline mesh. The effectiveness of AMR is further assessed by comparing the separation bubble which is caused by the interaction between strong shock wave and boundary layer, as given in Fig. 11. From the streamlines given in Fig. 11(a) and Fig. 11(b), the flow separation is triggered at the

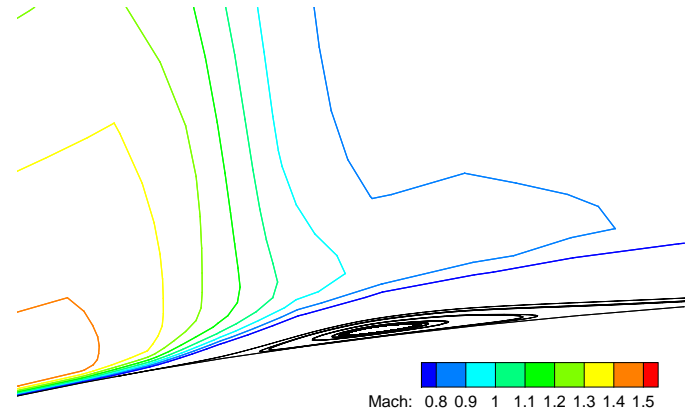

(a) Baseline mesh

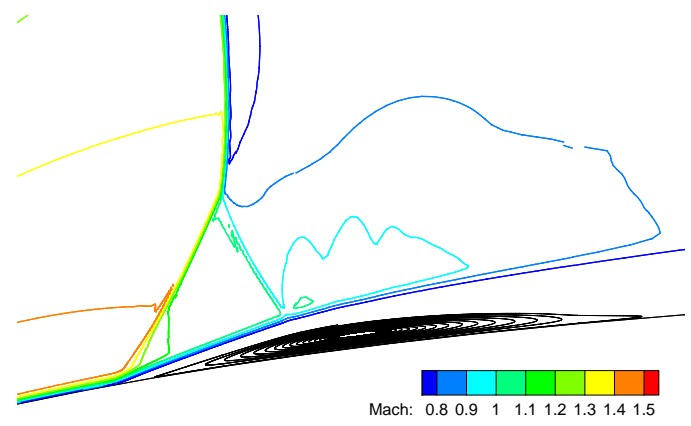

(b) 5 levels of AMR

Fig. 11 Comparison of the shock induced separation bubble, NACA-0012 test case.

location where the shock wave hits the boundary layer. Because the strong front foot of the $\lambda$ shock is not captured at all, with the baseline mesh the separation starts more downstream and the separation region covers smaller area compared to the AMR results. Accurate computation of these phenomena is essential to the estimation of separation related loss and the results in Fig. 11(b) are more accurate. The surface $c_{p}$ distributions are also compared with the experiment (Holst, 1987), as given in Fig. 12. Differences exist mostly in the upper surface shock wave region and $c_{p}$ with adapted mesh in this region is in better agreement with the experiment.

At last, the computation cost is compared. The baseline mesh has about 16k cells and after 5 levels of mesh refinement, the number of cells is increased to about $135 \mathrm{k}$. If global mesh refinement is used, about $17 \mathrm{M}$ cells are required to get similar mesh resolution and a vast cost saving is obtained with current AMR strategy. 


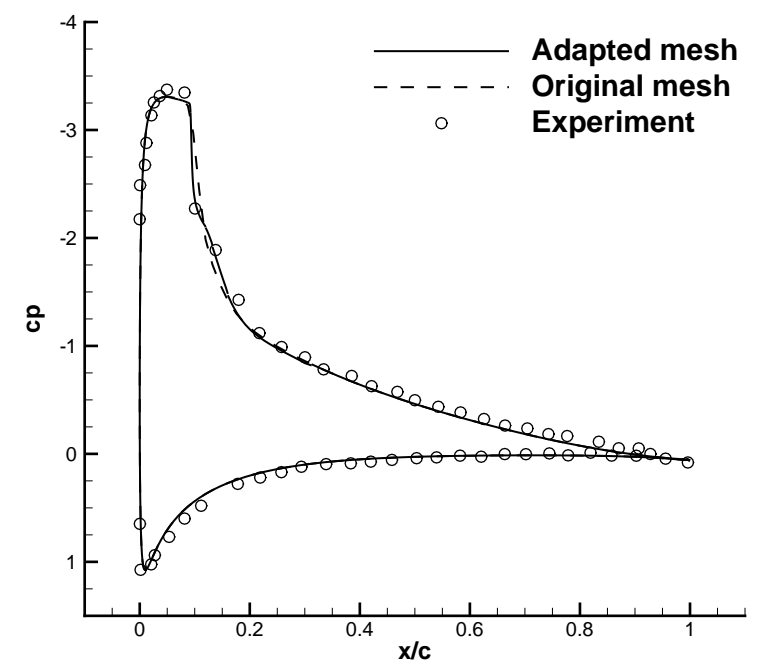

Fig. 12 Comparison of computed surface $c_{p}$ distributions with experiment, NACA-0012 test case.

\subsection{Flow past the Delta-wing}

In this example the subsonic flow past a three-dimensional delta-wing is computed. The freestream Mach number is 0.3 and strong vortical flow exists due to the high angle-of-attack of 20.5 degrees.

As in this case the flow is steady and thus half-model is computed. The baseline computational mesh is composed of $36 \times 64 \times 64$ cells. The geometry of this configuration is simple; however, it do proposes severe challenges to the body-fitted mesh based AMR because of several singular features, such as the singular point at the leading, the degenerated mesh line and the singular line on the edge of the wing, as given in Fig 13(b). If the original block based cubic interpolation is used to refine the mesh, almost all cells near these singular features have negative Jacobians. With current sub-block division algorithm, the baseline mesh block is automatically divided into 6 sub-blocks. Inside every sub-block the mesh lines are enough smooth to enable the generation of valid finer mesh, as shown in Fig. 13(c).

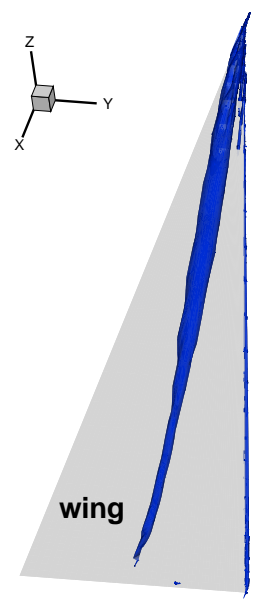

(a) Refinement criterion $\psi=0.01$ isosurface

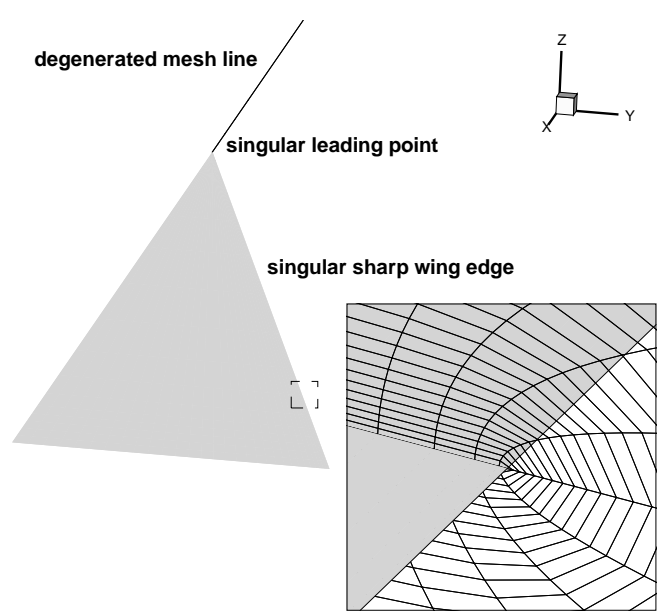

(b) Singular features of the mesh and the mesh lines around the sharp wing edge

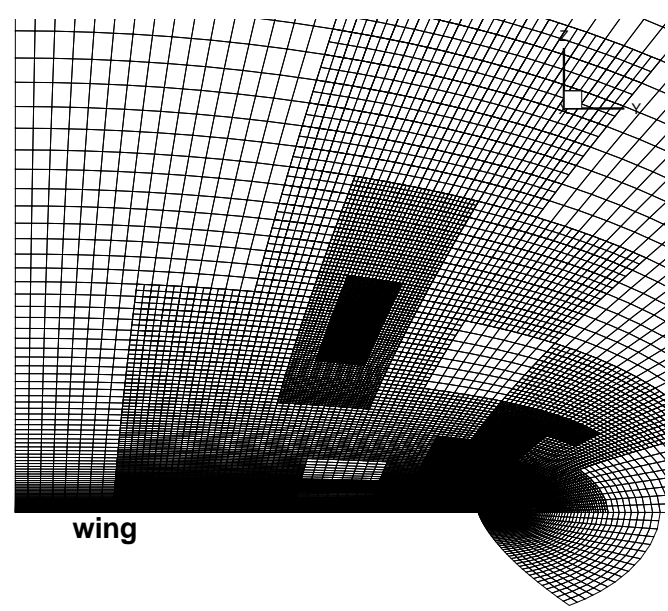

(c) Refined mesh at $x / L=0.5$ plane (the baseline mesh is not shown for clarity)

Fig. 13 Refinement criterion $\psi$ distribution and the AMR mesh, delta-wing test case.

This test case is shock-free and the primary interest is the accurate capturing of the strong vortical flow. For these types of flows currently the mostly used refinement criterion is vorticity based, such as the vorticity magnitude or the Q-criterion. Fig. 13(a) gives the $\psi=0.01$ isosurface. From Fig. 13 it is clear the function $\psi$ has a large value in the vortex core region and works well in marking the vortical region requiring higher resolution.

Due to the high angle-of-attack, a strong vortex is formed above the wing and there is an extremely low pressure region within the vortex core. Computed $c_{p}$ distributions with the baseline mesh are given in Fig. 14 and compared with the results after 4 levels AMR. Vortical flows, such as the aircraft wake and helicopter tip vortex, are important to the 


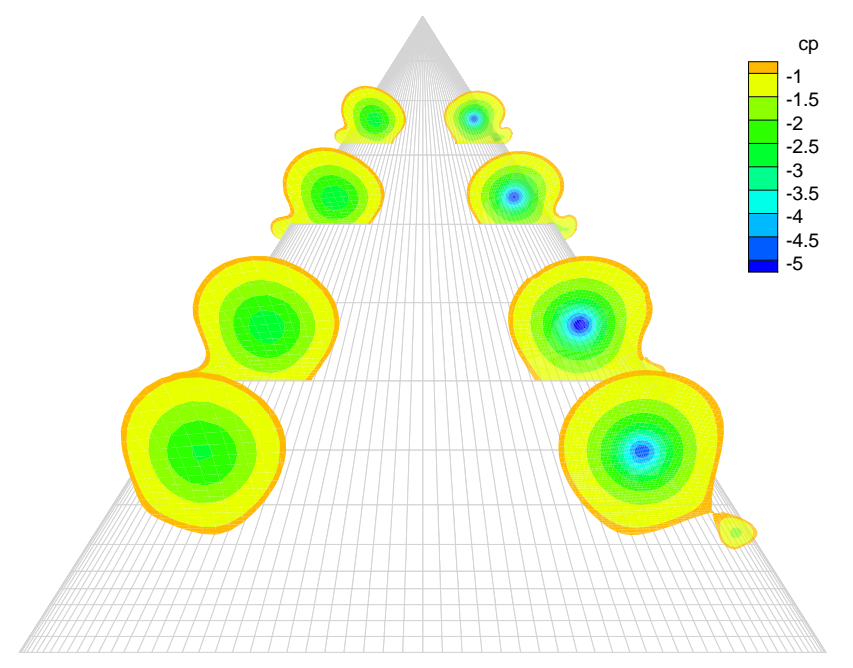

Fig. 14 Surface mesh points of the baseline mesh and $c_{p}$ distributions at four slices ranging from $x / L=0.2$ to $x / L=0.9$ of the delta-wing case. The left half denotes the results obtained using the baseline mesh and the right half represents the results after 4 levels of AMR.

performance of fluid machinery. Accurate capturing of the strong vortical flow needs high accuracy numerical scheme and fine mesh. With low accuracy scheme or insufficient mesh resolution, the vortex is always dissipated earlier and the local pressure minima is not well captured, as evidenced in Fig. 14. The numerical results in the vortex core region at the position of $x / L=0.5, z / L=0.0527$ are extracted and displayed in Fig. 15. From the convergence histories of $c_{p}$

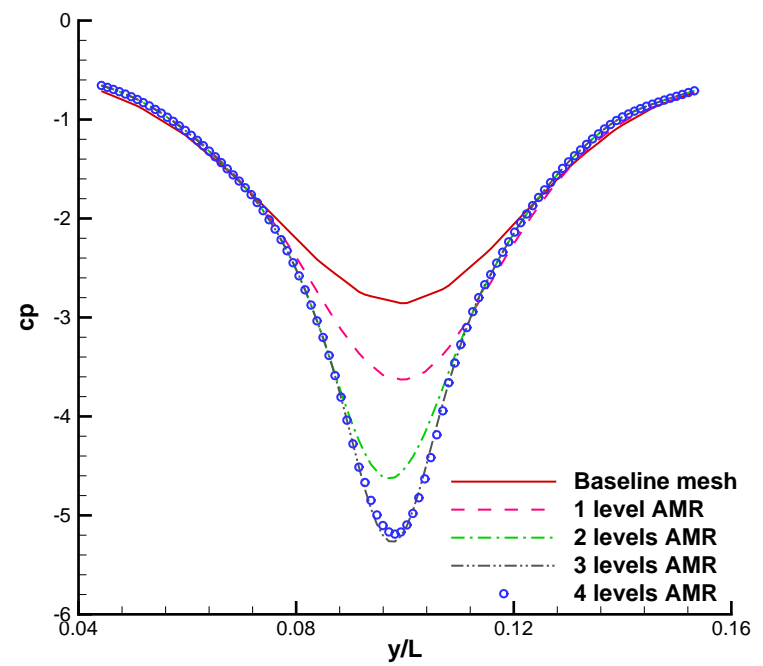

(a) Pressure coefficient $c_{p}$

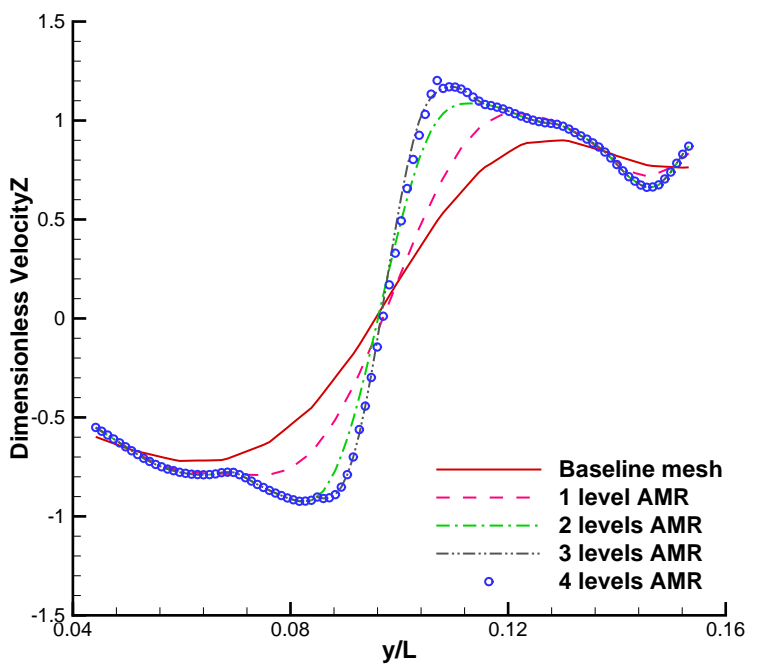

(b) Dimensionless vertical velocity $w / u_{\infty}$

Fig. 15 Plots of numerical results in the vortex center at position $x / L=0.5, z / L=0.0527$, delta-wing test case.

and $w / u_{\infty}$, it is clear after 4 levels adaption, mesh converged solution is obtained. For such kind of strong vortical flow, accurate capturing of the pressure minima proposes stringent requirement of mesh resolution, for example, from Fig. 15(a) with the baseline mesh the $c_{p}$ inside the vortex core is only a half of the converged value.

After 4 times adaption, the size of the mesh is increased by 45 times; however, if global mesh refinement is used, the mesh size will be increased by about 4000 times.

\subsection{Internal flow through a compressor stage}

In the above two external flow examples are given and here a test case about the internal flow through a transonic compressor stage will be discussed. Steady state computation is conducted and mixing plane is used between the rotor and stator. The computed relative Mach number distributions are given in Fig. 16(a). As demonstrated in Fig. 16(a), a shock wave of mild amplitude exists in the rotor passage. A large amount of loss is the consequence of shock wave and its interaction with boundary layer. The distributions of the refinement criterion are given in Fig. 16(b). The shock wave 


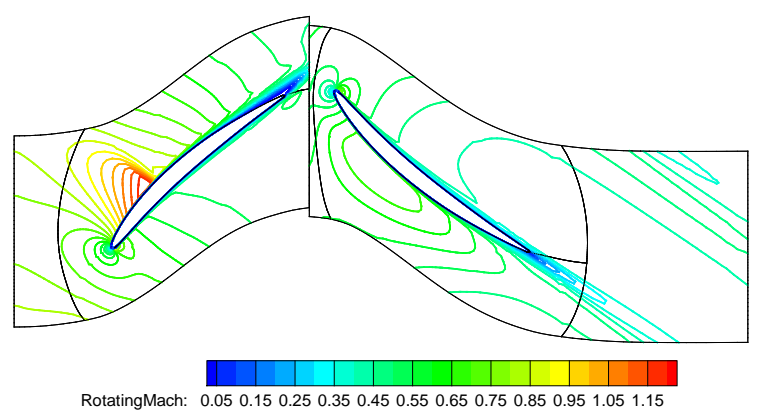

(a) Relative Mach number

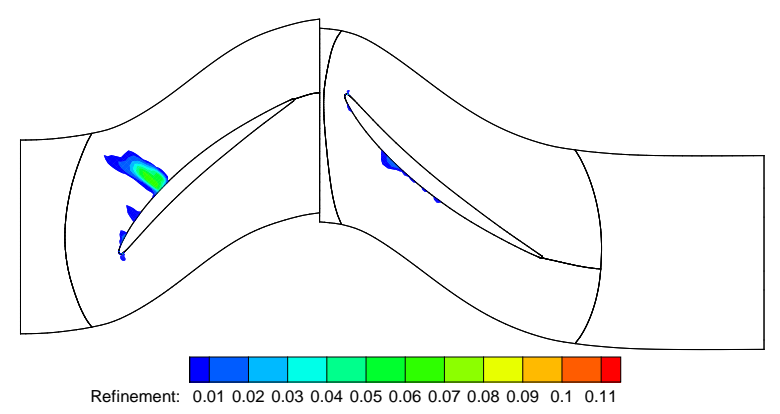

(b) Refinement criterion $\psi$

Fig. 16 Numerical results obtained with the baseline mesh, compressor stage case.

inside the rotor passage is automatically detected. Also near the leading edges of the rotor and stator, the resolution of the baseline mesh is based on rough estimation and does not provide enough resolution. This deficiency is automatically detected and the area near the leading edge is also marked for refinement. Current curvilinear AMR process is automatically conducted for 5 times and after 5 times refinement, the mesh size is increased by about 6 times. If the mesh is globally refined, the mesh size will be 1000 times larger and it is clear with AMR strategy a dramatic cost saving is obtained.

The results after 5 times refinement are given in Fig. 17. The computed results are further compared by checking

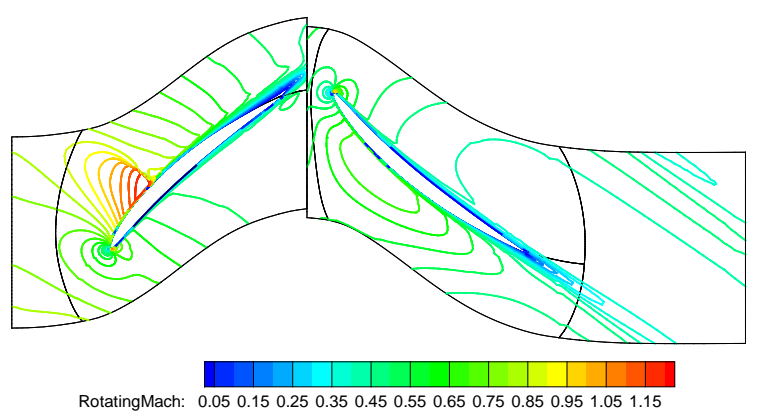

Fig. 17 Relative Mach number distributions after 5 times AMR refinement, compressor stage case.

the flow details, as given in Fig. 18. From Fig. 18(a), with the baseline mesh, the passage shock wave is monotonically

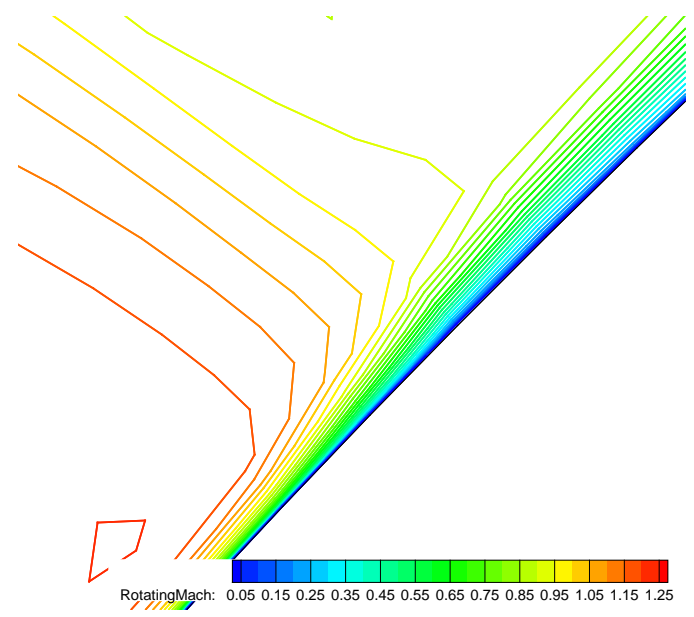

(a) Baseline mesh

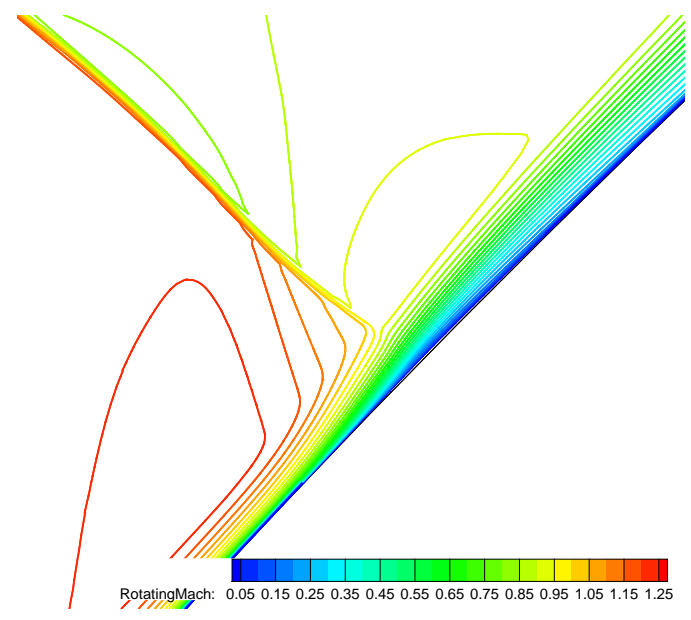

(b) AMR mesh after 5 times refinement

Fig. 18 Local relative Mach number distributions in the rotor passage, compressor stage case.

captured. The shock wave profile spans about two cells width and due to the large mesh size, the flow details are not well captured. For this test case, the maximum relative Mach number inside the rotor passage is about 1.25 and the shock wave is weaker than that in the NACA-0012 test case. At this Mach number, the interaction pattern between the shock wave and boundary layer changes and there is no shock wave induced flow separation. It is well agreed that the design of 
transonic rotor blade with high efficiency and wide operation range relies heavily on the proper organization of passage shock wave; however, the results from the baseline mesh do not provide enough details about it. The numerical results after 5 times AMR refinement is given in Fig. 18(b) and the interaction pattern is much clearer. From Fig. 18(b) a series of compression pressure waves exist near the boundary layer and a crisp normal shock wave exists in the passage. With the original mesh these details are totally lost.

The flow near the circular edge of the stator blade is also given here, as demonstrated in Fig. 19. The stator undergoes

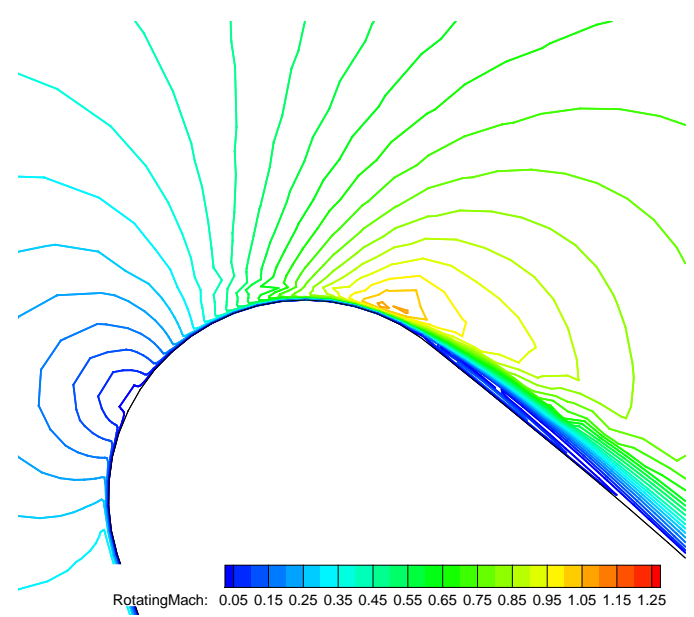

(a) Baseline mesh

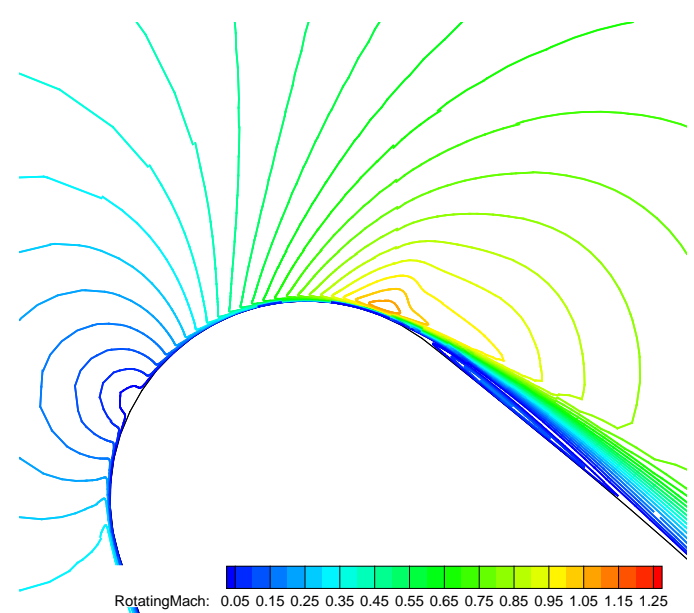

(b) AMR mesh after 5 times refinement

Fig. 19 Local Mach number distributions near the leading edge of the stator, compressor stage case.

a small positive incident angle. Caused by the incident angle, the flow undergoes large turning around the leading edge and it is rapidly accelerated to transonic and then decelerated to subsonic. The flow changes quickly in such a narrow space and the rough estimation based mesh density in the baseline mesh is obviously not suitable. Due to the insufficient resolution of the baseline mesh, there are obvious wiggles near the leading edge and these phenomena are unphysical. With the refined mesh, more mesh points are automatically positioned in this region and the local flow near the leading edge is much smoother, as given in Fig. 19(b).

\section{Conclusions}

In this work a body-fitted AMR tool is developed based on the BCM Cartesian mesh framework. For its efficient and robust applications with complex geometry, several key strategies are developed. A curvature based refinement function is used to detect both shock wave and strong vortical flow. To preserve the mesh qualities like smoothness and aspect ratio, cubic mappings are used to automatically refine the curvilinear mesh. For its applications with complex geometry where the singular geometry proposes severe challenges, a new refinement strategy is proposed where the mesh is automatically divided into sub-blocks. Then the cubic mesh refinement is conducted inside the sub-block and in this manner the accuracy and robustness issues are satisfied simultaneously. Combined with the high order WENO scheme, in this work a highly efficient and robust curvilinear mesh based AMR solver is developed. The applicability of current method is numerically verified and it works well with complex flow and is able to automatically capture flow details at much smaller cost compared to the global refinement strategy.

\section{Acknowledgements}

This work is supported by JSPS KAKENHI (21226018). The comments and suggestions from the anonymous reviewers are deeply appreciated. The drawback of the pressure curvature is kindly pointed out by one anonymous reviewer.

\section{References}

Berger, M. J., and Colella, P., Local adaptive mesh refinement for shock hydrodynamics, Journal of Computational Physics, Vol.82 No.1 (1989), pp.64-84. 
Berger, M. J., and Jameson, A., Automatic adaptive grid refinement for the Euler equations, AIAA Journal, Vol.23 No.4 (1985), pp.561-568.

Berger, M. J., and Oliger, J., Adaptive mesh refinement for hyperbolic partial differential equations, Journal of Computational Physics, Vol.53 No.3 (1984), pp.484-512.

Berger, M. J., and Rigoutsos, I., An algorithm for point clustering and grid generation, IEEE Transactions on Systems, Man and Cybernetics, Vol.21 No.5 (1991), pp.1278-1286.

Buning, P. G., and Pulliam, T. H., Initial implementation of near-body grid adaption in OVERFLOW, 11th Symposium on Overset Composite Grid and Solution Technology, (2012).

Groth, C. P. T., Zuuew, D. L. D., Powell, K. G., Gombosi, T. I., and Stout, Q. F., A parallel solution-adaptive scheme for ideal magnetohydrodynamics, AIAA-1999-3273, (1999).

Holst, T. L., Viscous transonic airfoil workshop, AIAA-1987-1460, (1987).

Jameson, A., Schmidt, W., and Turkel, E., Numerical solution of the Euler equations by finite volume methods using Runge-Kutta time-stepping schemes, AIAA-1981-1259, (1981).

Jiao, X., and Narasimha, B., Identification of C1 and C2 discontinuities for Surface Meshes in CAD, Computer-Aided Design, Vol.40 No.2 (2008), pp.160-175.

Jouhaud, J. C., Montagnac, M., and Tourrette, L., A multigrid adaptive mesh refinement strategy for 3D aerodynamic design, International Journal for Numerical Methods in Fluids, Vol.47 No.5 (2005), pp.367-385.

Lekien, F., and Marsden, J., Tricubic interpolation in three dimensions, International Journal for Numerical Methods in Engineering, Vol.63 No.3 (2005), pp.455-471.

Leer, V. B., Towards the ultimate conservative difference scheme III. Upstream-centered finite-difference schemes for ideal compressible flow, Journal of Computational Physics, Vol.23 No.3 (1977), pp.263-275.

Liu, X., Osher, S., and Chan, T., Weighted Essentially Non-oscillatory schemes, Journal of Computational Physics, Vol.115 No.1 (1994), pp.200-212.

MacNeice, P., Olson, K. M., Mobarry, C., Fainchtein, R., and Packer, C., PARAMESH: A parallel adaptive mesh refinement community toolkit, Computer Physics Communications, Vol.126 No.3 (2000), pp.330-354.

Matsuo, Y., Kuwabara, T., and Nakamori, I., A parallel structured adaptive mesh refinement approach for complex turbulent shear flows, Journal of Fluid Science and Technology, Vol.7 No.3 (2012), pp.345-357.

Mavriplis, D. J., Revisiting the Least-Squares Procedure for Gradient Reconstruction on Unstructured Meshes, AIAA2003-3986, (2003).

Nakahashi, K., High density mesh flow computations with Pre-/Post-data compressions, AIAA-2005-4876, (2005).

Nakahashi, K. Kitoh, A., Sakurai, Y. and Meinke, M., Three dimensional flow computations around an airfoil by BuildingCube Method, AIAA-2006-1104, (2006).

Spalart. P, and Allmaras. S. A., A one equation turbulence model for aerodynamics flows, AIAA-1992-0439, (1992).

Su, X., Sasaki, D., and Nakahashi, K., Cartesian mesh with a novel hybrid WENO/meshless method for turbulent flow calculations, Computers and Fluids, Vol.84 No.0 (2013a), pp.69-86.

$\mathrm{Su}, \mathrm{X}$., Sasaki, D., and Nakahashi, K., On the efficient application of Weighted Essentially Nonoscillatory scheme, International Journal for Numerical Methods in Fluids, Vol.71 No.2 (2013b), pp.185-207.

Su, X., and Yamamoto, S., A new matrix dissipation model for central scheme, International Journal for Numerical Methods in Fluids, Vol.74 No.7 (2014), pp.494-513. 